\title{
Tactile Feedback Can Assist Vision During Mobile Interactions
}

\author{
Jerome Pasquero \\ Research In Motion, \\ 295 Phillip Street, Waterloo, Ontario, \\ Canada N2L 3W8 \\ jerome.pasquero@gmail.com
}

\author{
Vincent Hayward \\ UPMC Univ Paris 06 \\ ISIR \\ 75005 Paris, France \\ vincent.hayward@isir.upmc.fr
}

\begin{abstract}
We evaluated the use of rich tactile feedback in the task of scrolling through a long list of items. We used a hand-held device having a tactile transducer that could provide sensations with temporal and spatial content. These capabilities were put to use in an interaction metaphor where input and tactile feedback were tightly coupled. We measured timeto-target and error rates, but also measured the time spent by participants to look at the screen. We found a $28 \%$ decrease of reliance on vision when tactile feedback was enabled.
\end{abstract}

\section{Author Keywords}

Mobile Interaction, Tactile Feedback

\section{ACM Classification Keywords}

H.5.2 Information Interfaces and Presentation: User InterfaceHaptic I/O; H.1.2 Models and Principles: User/Machine Systems Human information processing

\section{General Terms}

Information Interfaces And Presentation, Human Factors

\section{INTRODUCTION}

Mobile devices provide interaction affordances that are inherently more restricted than those possible with desktop configurations $[3,6]$. Because of the lack of display space and reduced input options, users of mobile devices are confronted with the attention-demanding process of navigating between multiples layers of menus in the interface. Interruptions from the environment complicate this task further. Providing tactile feedback to support navigation has the potential to reduce user disorientation and the amount of visual attention that must be devoted to the device. We considered the task of locating an item in a long list using a mobile device. Tasks of that type arise when searching for a correspondent in a list of contacts or looking for a particular tune in a portable music player, see Fig. 1(b).

\footnotetext{
Permission to make digital or hard copies of all or part of this work for personal or classroom use is granted without fee provided that copies are not made or distributed for profit or commercial advantage and that copies bear this notice and the full citation on the first page. To copy otherwise, or republish, to post on servers or to redistribute to lists, requires prior specific permission and/or a fee.

Proceedings of CHI 2011, pp. 3277-3280.

Copyright 2011 ACM 978-1-4503-0267-8/11/05...\$5.00.
}

We took advantage of a platform for the design and evaluation of application concepts which combine graphical and tactile feedback, the ТнМв, fully described elsewhere [12].
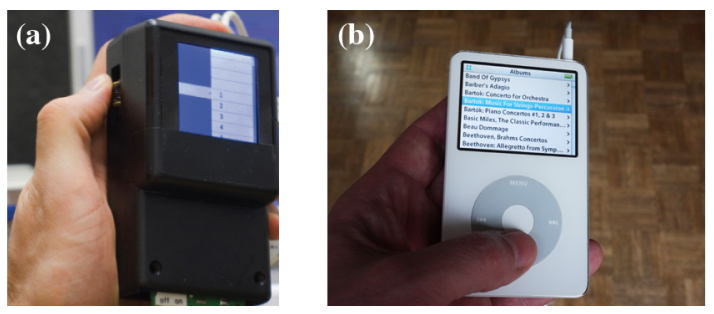

Figure 1. Itemized lists. (a) Тнмв device. (b) mobile music player.

The THMB's main component, shown in Fig. 1(a), is a handheld device prototype that can provide rich tactile feedback to the thumb in the form of spatiotemporal stimuli that encode time, location, speed, direction and intensity. Stimuli are created by using an array of piezoelectric benders that cause distributed lateral deformation in the skin. Using this technique, it is possible to convey sensations of fixed shapes (e.g. Braille dots) and moving features under the fingerpad $[7,8]$.

\section{RELATED WORK}

Research in multimodal interaction has long regarded tactile feedback as an indispensable channel. As early as in the 1960's, vibrotactile feedback was proposed to aid jet pilots to become aware of impending stalls [4]. Since then, it has been shown that when used in combination with vision in an interface, touch can provide added value in the form of an enhanced experience and/or a measurable increase in performance [2]. Advances in the miniaturization of actuators and progress in understanding of the tactile modality have sparked research intended to leverage touch in mobile interactions. Vibrotactile feedback has been shown to be effective at conveying abstract information by means of short tactile messages [1,9]. Other possibilities beyond a role as an alerting mechanism have also emerged. Poupyrev et al., and Oakley et al., have studied the effects of providing vibrotactile feedback during a scrolling task that requires participants to tilt a device for position and rate control [10, 13]. Similarly, Hoggan et al. demonstrated that the introduction of programmable tactile feedback to a text entry task on a touchscreen helps reduce the number of typing errors [5]. 


\section{EXPERIMENTAL PLATFORM}

The THMB comprised a tactile display that was co-located with a liquid crystal display to enable the coincidental display of graphical and tactile information. The present prototype included enhancements suggested by the results of perceptual characterization [8], including an increase in the strength of the tactile sensations. This was achieved by a mechanical redesign of the slider and changes to the electronics that made it possible to achieve a $800-\mathrm{Hz}$ refresh rate.

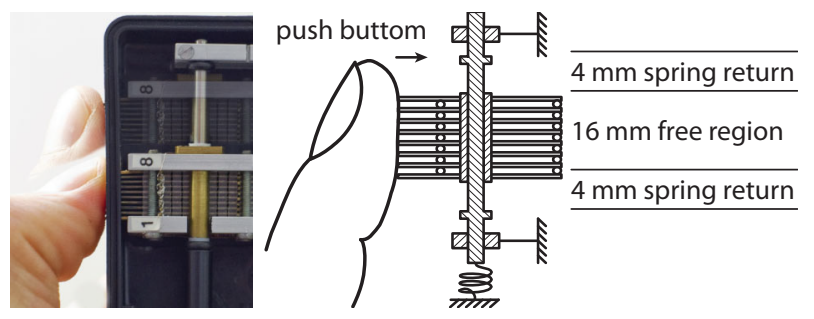

Figure 2. The tactile transducer was mounted on sliding mechanism that is spring-loaded and that acts as a push-button.

The resulting tactile transducer protruded slightly from a rectangular opening located under the thumb. It was mounted on a slider so it could be operated with a flexion of the thumb, see Fig. 2. The arrangement also acted as a pushbutton when pressed in the direction normal to the sliding axis. The slider was spring-loaded, which provided a restoring force to the central region. This new feature helped to locate the transducer inside the slider range. The range was divided into three distinct regions. The top and bottom regions were spring-actuated, while the central neutral region was free.

The tactile transducer had an array of eight $0.5 \mathrm{~mm}$-thick piezoelectric benders activated by modulating the voltage across their electrodes. Tactile stimulation was provided by programming the benders to cause tangential traction at the surface of the skin, over an active surface of $10 \times 8 \mathrm{~mm}$, a process that we term 'laterotactile stimulation'.

\section{DESIGN OF A FINE-POSITIONING CONTROLLER}

We designed a control metaphor that was well suited to the precise scrolling within large lists. A first group of 8 persons was recruited to participate in an iterative design process comprising a cycle of short informal evaluation sessions and calibration sessions. During calibration sessions, participants operated the THMB device by trying different mappings during a scrolling task, and described their experience to the designer. These comments were instantly addressed by using an elaborate interactive waveform editor in order to adjust control parameters such as intensities and gains.

The resulting controller provided three scrolling modes that corresponded to the three different regions: a stop mode, a discrete mode and a continuous mode. When the transducer was in the central, free region, the list was static. A slight push against one of the boundaries triggered the discrete mode which caused the list to scroll by exactly one item, like with a scroll wheel. Pushing further into the spring-return region switched the controller to a continuous mode where
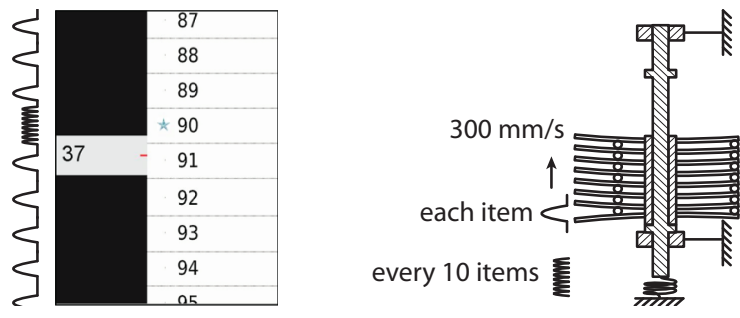

Figure 3. Metaphor for the experiment: a list of 100 numeric items marked by tactile bumps. One in 10 elements was marked with a highfrequency texture. Tactile animations traveled across the tactile display in the direction opposite to the scrolling motion.

the list scrolled with an acceleration that was proportional to the amount of deflection. Acceleration and terminal velocity were programmed not to exceed 3 item $/ \mathrm{s}^{2}$ and 12 items/s, respectively. Finally, when the button was released, the spring returned the transducer back to the neutral zone where the list was brought to rest.

Tactile feedback was provided in the form of two highly distinguishable tactile icons: a short traveling wave was triggered for each list item and a longer wave of higher spatial frequency indicated the passing of 10 items, as illustrated in Fig. 3. Waves traveled against the scrolling motion of the list to reinforce the sensation of sliding over items as they passed by. When not activated, the piezoelectric actuators were programmed to bend towards the top, see Fig. 3. As a tactile icon passed by, each actuator exhibited the same full back-and-forth bending motion, but with a phase difference. A traveling speed of $300 \mathrm{~mm} / \mathrm{s}$ was selected because it combined the advantages of speed and efficiency at delivering directional cues [8]. The flow of tactile sensations under the thumb evoked equally spaced bumps on a road. In the absence of visual information, it was therefore possible to experience the scrolling speed from the stimulus frequency and traveling wave group velocity.

\section{EXPERIMENT}

To probe the participants' behaviors, we recorded the frequency at which they needed to look at the screen to perform the task. We employed a very simple testing method. By default, the list was not visible. Each time the participants felt that they needed visual information, they had to press and hold down a key of the computer to make it visible. The scrolling list was made of the numbers 1 to 100. By recording the number of keystrokes and the manner in which participants moved inside the list, we could paint an accurate picture of their behavior under different testing conditions.

\section{Methods}

\section{Sessions}

Experimental sessions were divided into three blocks of trials that corresponded to three different conditions: a training condition, a control condition, and a condition with tactile feedback. The training condition was always administered first and consisted of an informal session during which participants could get familiar with the task. It also allowed the experimenter to ensure that participants were performing the 
task at a pace compatible with a natural range of operation. The experimental task was the same for all three conditions. For the training and the control conditions, the tactile feedback generated by the controller was disabled. Half of the participants were tested in the control condition before the tactile condition, and the other half in reverse order.

\section{Participants}

Ten graduate students between 25 and 34 years of age (mean 28) were recruited for this experiment. They were compensated $\$ 10$ for their participation that lasted about 45 minutes. All participants, but one, were right-handed. None had participated in the previous phase of calibration of the device.

\section{Task}

Participants were instructed to scroll to a target and to select it. The list was made of 100 consecutive integers and was invisible by default. To view the list, the participants had to press and hold down a key. When they released it, the graphical display was turned off. They were instructed to minimize the number of viewings and their duration. The target number was displayed to the left of the list and was considered to be reached when it was aligned with a horizontal cursor located at the center of the screen as in Fig. 3. Once the target was reached, subjects pressed the push-button to move to the next trial. Off-target button clicks were recorded but a trial was not over until the correct target was selected.

\section{Design}

The blocks of trials under the control and tactile conditions were exactly the same but the presentation order of the trials were randomized. A trial was defined by an initial index position and a target position that the subject needed to reach. Pairs of positions were separated by both short distances $(5$, 8, 10 items) and long distances (30, 40, 50 items) in order to be representative of all typical scrolling interactions. Each of the six distances was presented four times, for a total of 24 trials per condition. Half of the trajectories required subjects to scroll upwards and the other half required them to scroll in the opposite direction. Starting positions were randomized.

\section{Results}

We analyzed the difference between the control and tactile conditions for a number of dependent variables, see Table 1. Analysis using the Wilcox matched-pairs, signed-rank test revealed a significant difference between the control and tactile conditions in the number of key strokes (associated to visual glances), the time between two key strokes (switchback durations), and the number of overshoots. As shown on fig. 4, the number of viewings required to reach the target decreased by $28 \%$ when tactile feedback was enabled $(p<0.05)$. Closer analysis revealed that the reduction of the number of glances was more prominent over the longer scrolling distances (30, 40 and 50 items). The mean task completion time (i.e. the time required to reach all 24 targets) did not differ in a statistically significant way between the two experimental conditions; this also holds true when short and long distances are analyzed separately. Hence, tactile feedback did not slow down the scrolling task. Error rates, or the number of times participants clicked the wrong target, dropped by more than $50 \%$, but this was not found to be statistically significant. For all dependent variables, there was no difference between scrolling up and scrolling down. All ten participants could reduce their dependance on graphic information when tactile feedback was provided. However, the decrease in the number of glances was not distributed evenly, ranging from a $2.5 \%$ drop to a $49 \%$ drop.

Table 1. Task means. Bonferroni-corrected statistically significant differences are in bold; standard deviations are in parentheses.

\begin{tabular}{lrr}
\hline Independent Variable & Control & Tactile Feedback \\
\hline completion time (s) & $373.2(103.2)$ & $394.8(143.9)$ \\
visible proportion $(\%)$ & $7.8(5.7)$ & $6.0(5.0)$ \\
nb. of clicks off target & $14.0(12.1)$ & $6.9(4.4)$ \\
number of glances & $\mathbf{9 7 . 6}(31.9)$ & $\mathbf{6 9 . 8}(16.6)$ \\
switch-back duration (s) & $\mathbf{4 . 1}(1.3)$ & $\mathbf{6 . 3}(4.2)$ \\
number of overshoots & $\mathbf{9 . 3}(4.0)$ & $\mathbf{1 4 . 2}(5.0)$
\end{tabular}

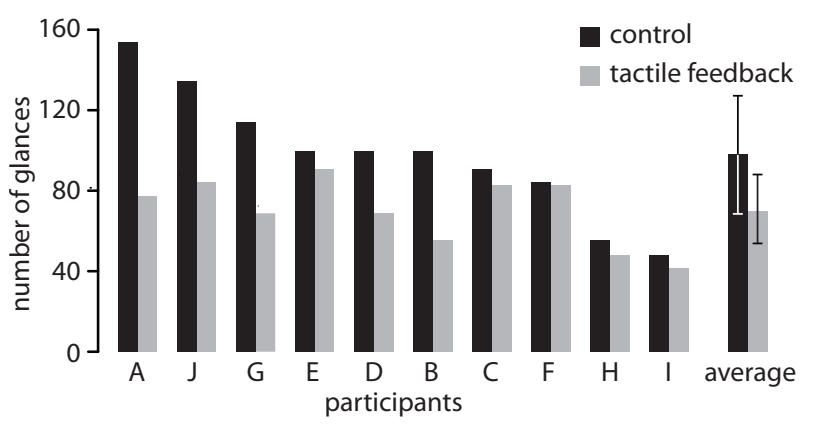

Figure 4. Number of glances at the screen.

The effect of tactile feedback was also seen in an increase in the switch-back duration, defined as the average time spent attending to the environment, modelled here as an invisible viewing window $(p<0.05)$. Switch-back durations collected correspond closely to those observed in a semi-natural field study during simple mobile interactions [11]. Results also show a increase of $52 \%$ in the number of overshoots when tactile feedback was introduced $(p<0.05)$.

Inspection of the trajectory profiles gave information regarding the different scrolling strategies employed by the participants. Fig. 5 shows samples of trajectories that indicate how the overall number of glances was reduced and why there was an increase in the number of overshoots. In all four cases, the participants' reliance on visual input was reduced. Fig. 5(a)-(d) illustrate how participants used more than one strategy, sometimes making use of the discrete scrolling mode alone, and in other times combining it with the continuous mode to guarantee a smooth landing on the target. Upon interview, most participants reported that they would first try to reach a position close to the target in continuous scrolling mode, then briefly glance at the screen before a final adjustment in discrete mode. Sometimes, however, they would initially underestimate the scrolling speed and end up overshooting the target, as it is the case for the sample trajectory in Fig. 5(d). Nevertheless, such errors were typically rectified promptly, with little consequences on the overall time needed to reach the target and without the need for any ex- 


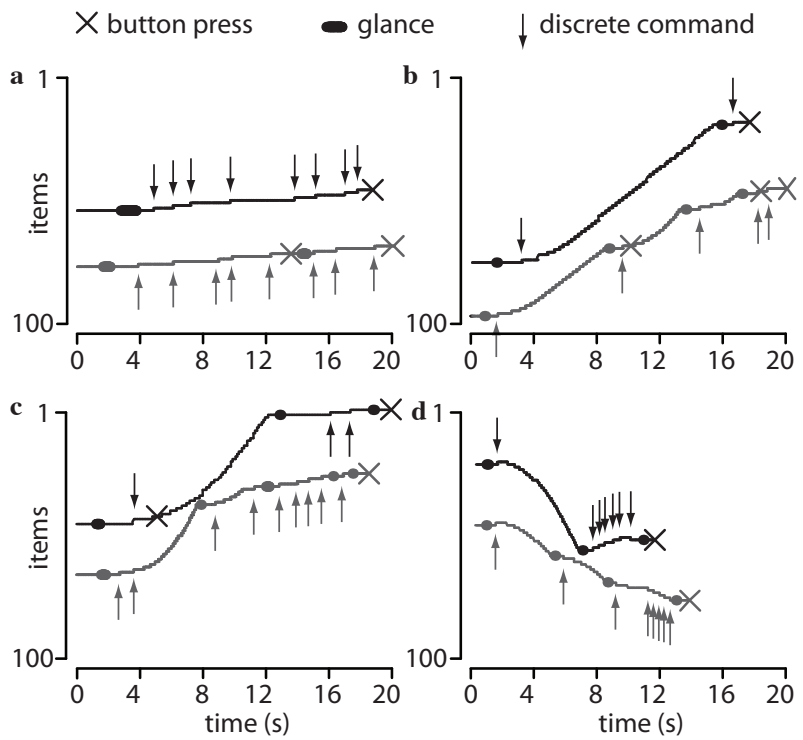

Figure 5. Trajectory profiles showing examples of where the number of screen glances are reduced. Tactile feedback condition is shown in black and the control in grey. The trajectories are shifted for clarity.

tra glance at the screen. The higher number of overshoots can be explained by the fact that tactile feedback allowed some participants to adopt an efficient scroll-and-recovery strategy for which an overshoot carried little penalty in task completion time.

\section{DISCUSSION AND CONCLUSION}

When tactile feedback was disabled, participants relied on a mental model that they had constructed during previous phases of the experiment. They used glances to recalibrate this model and navigated by dead reckoning between glances. The results suggest that directional tactile cues were well mapped to the control inputs and perhaps enabled participants to anticipate the sensory consequences of their motor output. Tactile cues allowed them to adjust their scrolling strategies and to reduce the frequency at which they felt the need to look at the screen. This function resulted from a careful design of the tactile feedback provided in response to input commands. By contrast, existing tactile feedback technologies for mobile devices, such as vibration motors, cannot reproduce the sensation of a fast moving pulse and cannot be activated fast enough to provide a sensation that evokes the dash line of a road at high scrolling speeds.

We ran an experiment that estimated the amount of visual attention devoted to the interaction simply by asking the participant to have control over it. Results are encouraging. The interaction metaphor that we used led to an average reduction of $28 \%$ in the number of glances that the participants required to complete the task without any effect on task time. The benefits introduced were not evenly distributed across the participants, but they all reacted positively to the tactile feedback. This suggests a new unexplored role for tactile feedback and shows how it can assist the visual modality in ways that go beyond improvements measured with typical evaluation metrics such as time-to-target and error rates.

\section{ACKNOWLEDGEMENTS}

The research was entirely conducted at the McGill University Haptics Lab, Canada. Funding was provided by a grant from the Natural Sciences and Engineering Council of Canada. The authors are grateful to Vincent Levesque and Prasun Lala for invaluable comments.

\section{REFERENCES}

1. S. A. Brewster and L. M. Brown. Tactons: Structured tactile messages for non-visual information display. In Proc. AUIC'04, pages 15-23, 2004.

2. J. L. Burke, M. S. Prewett, A. A. Gray, L. Yang, F. R. B. Stilson, M. D. Coovert, L. R. Elliot, and E. Redden. Comparing the effects of visual-auditory and visual-tactile feedback on user performance: a meta-analysis. In Proc. ICMI '06, pages 108-117, 2006.

3. L. Chittaro. Visualizing information on mobile devices. Computer, 39(3):40-45, 2006.

4. R. H. Cook. An automatic stall prevention control for supersonic fighter aircraft. Journal of Aircraft, 2(3):171-175, 1965.

5. E. Hoggan, S. A. Brewster, and J. Johnston. Investigating the effectiveness of tactile feedback for mobile touchscreens. In Proc. CHI'08, pages 1573-1582, 2008.

6. S. Jones, M. Jones, G. Marsden, D. Patel, and A.Cockburn. An evaluation of integrated zooming and scrolling on small screens. Int. J. Human-Computer Studies, 63(3):271-303, 2005.

7. V. Lévesque, J. Pasquero, V. Hayward, and M. Legault. Display of virtual braille dots by lateral skin deformation: feasibility study. ACM T. Applied Perception, 2(2):132-149, 2005.

8. J. Luk, J. Pasquero, S. Little, K. MacLean, V. Levesque, and V. Hayward. A role for haptics in mobile interaction: initial design using a handheld tactile display prototype. In CHI 'O6, pages 171-180, 2006.

9. K. MacLean and M. Enriquez. Perceptual design of haptic icons. In Proc. Eurohaptics, 2003.

10. I. Oakley and S. O’Modhrain. Tilt to scroll: Evaluating a motion based vibrotactile mobile interface. In Proc. WHC '05, pages 40-49, 2005.

11. A. Oulasvirta, S. Tamminen, V. Roto, and J. Kuorelahti. Interaction in 4-second bursts: the fragmented nature of attentional resources in mobile HCI. In Proc. CHI '05, pages 919-928, 2005.

12. J. Pasquero, J. Luk, V. Levesque, Q. Wang, V. Hayward, and K. E. MacLean. Haptically enabled handheld information display with distributed tactile transducer. IEEE T. on Multimedia, 9(4):746-753, 2007.

13. I. Poupyrev, S. Maruyama, and J. Rekimoto. Ambient touch: designing tactile interfaces for handheld devices. In Proc. UIST '02, pages 51-60, 2002. 